\title{
Intrahepatic Cholangiocarcinoma with Ductal Plate Malformation-like Feature Associated with Bile Duct Adenoma
}

\author{
Ah-Young Kwon · Hye Jin Lee · Hee Jung An · Haeyoun Kang · Jin-Hyung Heo · Gwangil Kim \\ Department of Pathology, CHA Bundang Medical Center, CHA University, Seongnam, Korea
}

Intrahepatic cholangiocarcinoma (ICC) is a malignant tumor with biliary epithelial differentiation. Malignant transformation of von Meyenburg complex (VMC) to ICC has been reported, ${ }^{1,2}$ and a new subtype of ICC with ductal plate malformation (DPM) pattern has been suggested. ${ }^{3}$ However, bile duct adenoma (BDA) is a rare entity and is not as well known as DPM. Moreover, it has not been determined whether BDA is a risk factor of ICC. We present a rare case of ICC with DPM-like features associated with BDA.

\section{CASE REPORT}

The publication of the case information and materials was approved by the Institutional Review Board of CHA Bundang Medical Center, CHA University.

A 34-year-old female patient was referred for further evaluation of a small hepatic nodule found on a regular health checkup. She did not have any remarkable medical history associated with liver disease. On magnetic resonance imaging, a 2-cmsized mass was present in liver segment 4, showing high signal on T1- and low signal on T2-weighted images (Fig. 1A).

The patient underwent hepatic segmentectomy. The liver showed a relatively well-demarcated, subcapsular $(5 \mathrm{~mm}$ from the capsule), nonencapsulated, solid, rubbery, and pale brown mass. It was multilobulated with a central fibrous scar (Fig. 1B).

Histologically, the nodule was composed of three distinct ar-

\section{Corresponding Author}

Gwangil Kim, MD

Department of Pathology, CHA Bundang Medical Center, CHA University, 59 Yatap-ro,

Bundang-gu, Seongnam 13496, Korea

Tel: +82-31-780-5452, Fax: +82-31-780-5476, E-mail: blacknw@cha.ac.kr

Received: March 24, 2014 Revised: May 10, 2015

Accepted: June 19, 2015 eas. First, many compact, small, tubular structures lined by single cuboidal to low columnar epithelial cells were present without bile or dilated ducts. Nuclei were small and uniform without any mitotic activity, which was compatible with BDA containing portal tracts (Fig. 2A). Second, the central area showed DPMlike features, having irregularly dilated ductal structures lined by low columnar neoplastic epithelial cells with mild pleomorphism within fibrous stroma (Fig. 2B). Third, the opposite side of the BDA showed ICC. Columnar to cuboidal epithelial cells forming fused glandular structures with nuclear anaplasia and frequent mitoses were present (Fig. 2C). There were transitional areas from BDA to ICC (Fig. 2D).

On immunohistochemistry, cytokeratin (CK) 7, CK19, and epithelial cellular adhesion molecule (EpCAM) were positive, and monoclonal carcinoembryonic antigen (CEA), CD117, p53, and hepatocyte antigen were negative in all three areas. The ICC area showed diffuse positivity for polyclonal CEA; in contrast, the BDA and DPM-like areas showed apical reactivity only. Epithelial membrane antigen was negative in the BDA area, apically reactive in the DPM-like area, and strongly reactive in the ICC area. NCAM was positive in the ICC area, focally positive in the DPM area, but negative in the BDA area. The Ki-67 labeling index was variable, with values of $1 \%-2 \%$ in the BDA area, 10\%-20\% in the DPM-like area, and 40\%-50\% in the ICC area (Table 1, Fig. 3).

The remaining parenchyme did not show VMC or DPM features. No recurrence or metastasis was observed at a 28 -month follow-up.

\section{DISCUSSION}

Some benign hepatic biliary lesions, such as VMC or bile duct 

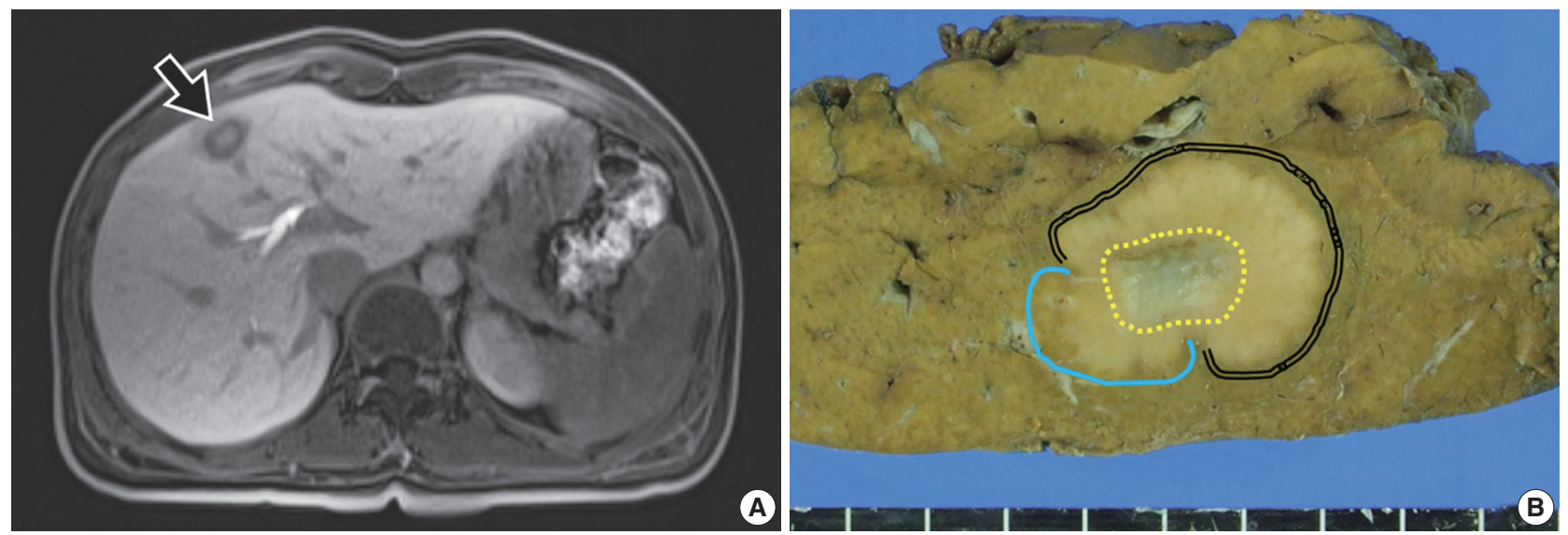

Fig. 1. Radiologic and gross findings. (A) Magnetic resonance imaging of the liver reveals a 2-cm target appearance lesion (arrow) in segment 4. On a T1-weighted image, the central portion shows low signal intensity (SI), and the peripheral zone shows intermediate to slightly high SI. (B) Grossly, the tumor is a relatively well-defined, solid, pale brown mass with a multinodular margin and central fibrous scar. The tumor has three areas: double line of right upper area, cholangiocarcinoma; dotted central circle, dilated ducts with fibrous stroma; and line of left lower area, bile duct adenoma.
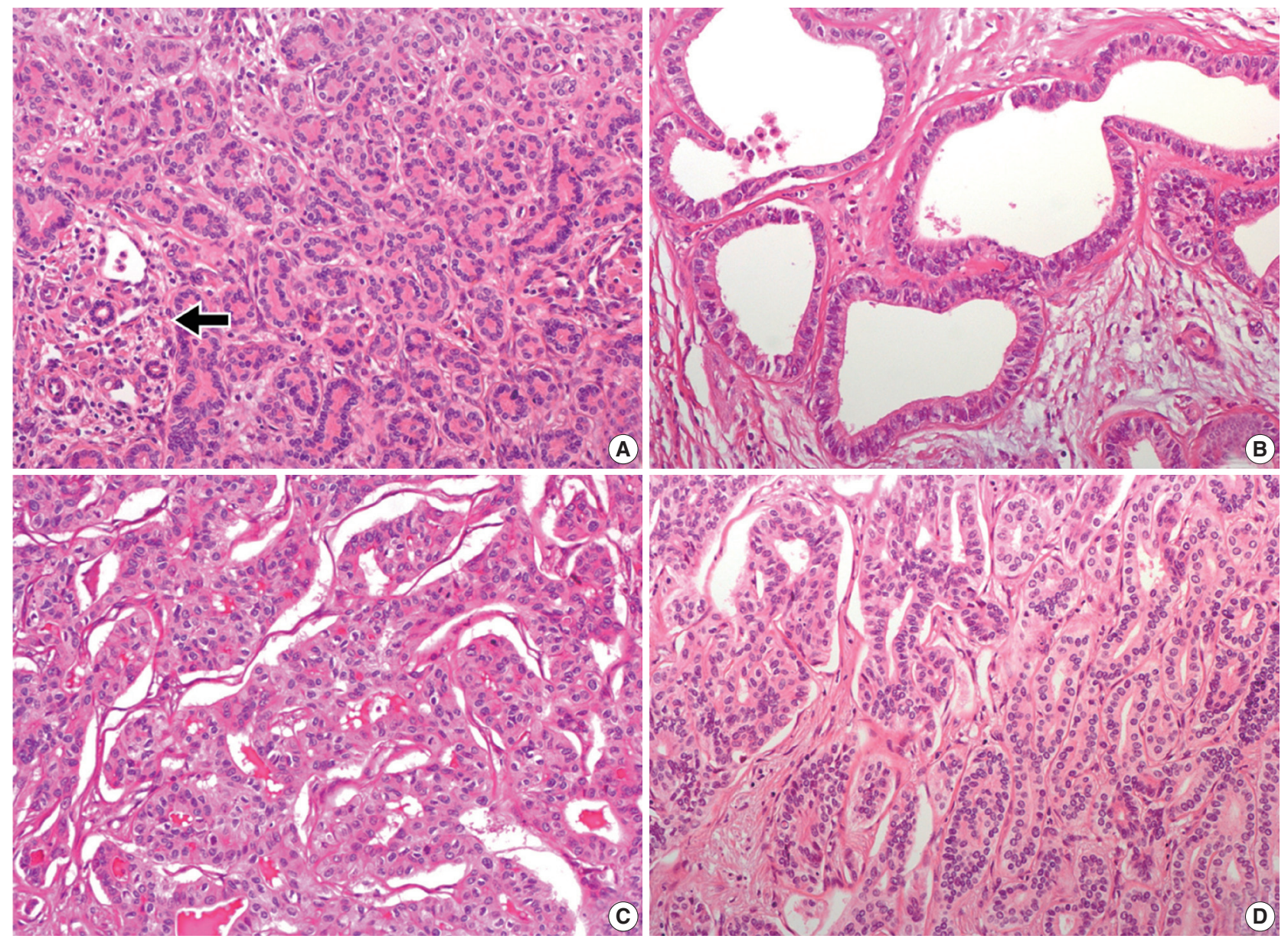

Fig. 2. Microscopic findings of the tumor. (A) One peripheral portion shows highly packed ducts with bland looking nuclei; bile duct adenoma containing portal tracts (arrow). (B) Central area reveals irregularly dilated glandular structures within fibrous stroma, resembling features of ductal plate malformation. (C) In the other peripheral lesion, fused and cribriform glands infiltrate into the stroma. The nuclei are atypical and show brisk mitotic activity; cholangiocarcinoma. (D) The tumor shows a transitional area between bile duct adenoma (right) and cholangiocarcinoma (left). Bland uniform ductal structures become irregular and anastomosing. 
adenofibroma, are known candidate precursors of ICC. ${ }^{4} \mathrm{VMC}$ is a congenital anomaly of biliary cells forming a hepatic tumorlike lesion. ${ }^{5}$ Intrahepatic cholangiocarcinoma arising in VMC has been observed since 1961., According to the ductal plate hypothesis proposed in 2011, ${ }^{7}$ VMC is implicated in DPM as a developmental anomaly of fetal biliary cells (ductal plate). Re-

Table 1. Immunohistochemical stain of tumor

\begin{tabular}{llccc}
\hline Antigen & Source, clone & BDA area & DPM area & ICC area \\
\hline CK7 & Neomarker, OV-TL 12/30 & $\mathrm{P}$ & $\mathrm{P}$ & $\mathrm{P}$ \\
CK19 & Neomarker, A53-B/A2.26 & $\mathrm{P}$ & $\mathrm{P}$ (apical) & $\mathrm{P}$ (membranous) \\
Polyclonal CEA & Neomarker, CEA Ab-2 & $\mathrm{N}$ & $\mathrm{N}$ & $\mathrm{N}$ \\
Monoclonal CEA & Neomarker, COL1 & $\mathrm{P}$ & $\mathrm{P}$ & $\mathrm{P}$ \\
EpCAM & Novocastra, VU-1D9 & $\mathrm{N}$ & $\mathrm{P}$ (apical) & $\mathrm{P}$ (membranous) \\
EMA & Cell MARQUE, E29 & $\mathrm{N}$ & $\mathrm{P}$ \\
NCAM (CD56) & Roche, 123C3 & $\mathrm{N}$ & $\mathrm{N}$ (focal) & $\mathrm{N}$ \\
CD117 (c-Kit) & DAKO, rabbit polyclonal & $\mathrm{N}$ & $\mathrm{N}$ & $\mathrm{N}$ \\
P53 & DAKO, DO-7 & $\mathrm{N}$ & $\mathrm{N}$ & $\mathrm{N}$ \\
Hepatocyte & DAKO, OCH1E5 & $1-2$ & $10-20$ & $40-50$ \\
Ki-67 (\%) & Neomarker, SP6 & & \\
\hline
\end{tabular}

BDA, bile duct adenoma; DPM, ductal plate malfromation; ICC, intrahepatic cholangiocarcinoma; CK, cytokeratin; P, positive; CEA, carcinoembryonic antigen; $\mathrm{N}$, negative; EMA, epithelial membrane antigen.

Table 2. Cases of cholangiocarcinoma associated with bile duct adenoma

\begin{tabular}{|c|c|c|c|c|c|c|c|}
\hline Reference & Year & Sex/Age (yr) & Location & Size $(\mathrm{cm})$ & Operation & Histology & $\begin{array}{c}\text { Associated liver } \\
\text { disease }\end{array}$ \\
\hline Hasebe et al. ${ }^{8}$ & 1995 & $M / 59$ & S4 & 2.2 & Partial resection & ICC with BDA and VMC & No \\
\hline Takahashi et al. ${ }^{10}$ & 2010 & $\mathrm{M} / 76$ & S6 & 3 & Resection & ICC with BDA and VMC & No \\
\hline \multirow[t]{2}{*}{ Pinho et al. ${ }^{9}$} & 2012 & $\mathrm{~F} / 60$ & S5 & 3.83 & Liver biopsy (at the age of 58) & $\mathrm{BDA}$ & No \\
\hline & & & & & Right hepatectomy & ICC & \\
\hline Present case & 2015 & $F / 36$ & S4 & 2 & Segmentectomy & $\begin{array}{l}\text { ICC with DPM pattern } \\
\text { associated with BDA }\end{array}$ & No \\
\hline
\end{tabular}

ICC, intrahepatic cholangiocarcinoma; BDA, bile duct adenoma; VMC, von Meyenburg complex; DPM, ductal plate malformation.

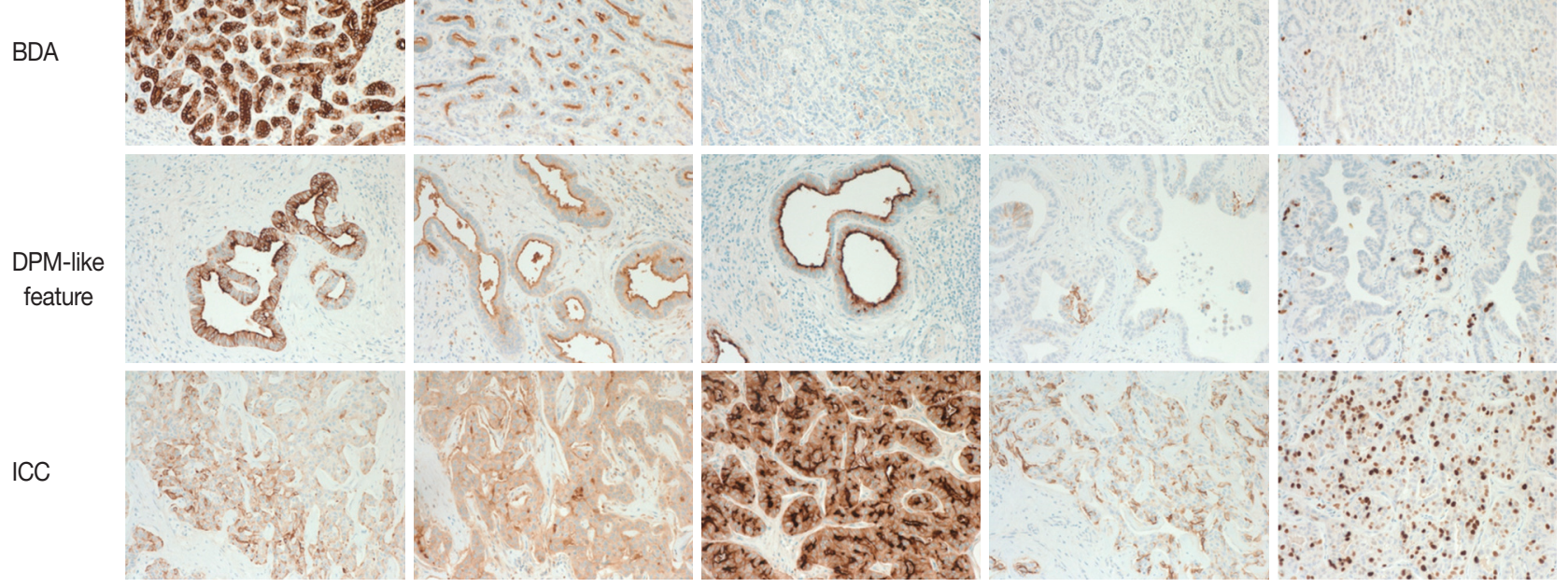

Fig. 3. Immunohistochemical staining patterns in three areas. Cytokerain 19 (CK19) and polyclonal carcinoembryonic antigen (CEA) are positive in all areas, but intensity and location are different. Epithelial membrane antigen (EMA) and NCAM are negative in the bile duct adenoma (BDA) area, weakly positive in the ductal plate malformation (DPM) area, and positive in the cholangiocarcinoma (ICC) area. The Ki-67 labeling index differs in the different areas, from $1 \%-2 \%$ in BDA to $40 \%-50 \%$ in the cholangiocarcinoma area. 
cently, cases of ICC with VMC features in a large proportion of the tumor are reported as ICC with predominant DPM pattern (ICC-DPM), a new subtype of ICC. ${ }^{3}$

In the present case, the tumor showed three histologically distinct areas of BDA, DPM, and ICC, and their proportions were $30 \%, 20 \%$, and $50 \%$, respectively.

$\mathrm{BDA}$ is a rare solitary intrahepatic lesion that consists of many small, uniform ducts with benign cuboidal cells and a narrow lumen. The BDA area in the present case was typical and localized to one side. Although BDA can be confused with bile ductular carcinoma foci of ICC-DPM, the latter show malignant epithelium and similar immunoreactivity to ICC-DPM. In contrast to VMC, BDA is not regarded as a precursor of ICC because ICC with BDA has been reported in only three cases (Table 2) ${ }^{8-10}$

DPM-like areas in our case revealed irregularly dilated glands within fibrous stroma, resembling VMC. The neoplastic columnar cells were different from typical VMC. This DPM-like feature might be a part of ICC-DPM or represent a transitional area between BDA and ICC. There were several unique points in the present DPM-like features that differ from the previously reported ICC-DPM. First, the typical irregular protrusions and bridging structures were not prominent in the DPM-like area in the present case. Second, there was no obvious stromal invasion in this area. Third, ICC and BDA in this case were distinguishable from the DPM-like area grossly, histologically, and immunohistochemically (especially with respect to CEA, EpCAM, NCAM, and Ki-67). ${ }^{3}$

The results of immunohistochemical staining of each area corresponded to the histological diagnosis. Intriguingly, NCAM was expressed in ICC and focally in the DPM-like area. This result supports the previous suggestion that ICC with DPM features is a subtype of hepatocellular-cholangiocarcinoma with stem cell features. ${ }^{5}$

In summary, we present a case of ICC with DPM-like features associated with BDA. Although the etiologic relationship between ICC and BDA or DPM needs further study, the possibility of BDA as a precursor of ICC is presented. Such a situation should be considered when BDA is found on a needle biopsy.

\section{Conflicts of Interest}

No potential conflict of interest relevant to this article was reported.

\section{REFERENCES}

1. Jain D, Nayak NC, Saigal S. Hepatocellular carcinoma arising in association with von-Meyenburg's complexes: an incidental finding or precursor lesions? A clinicopatholigic study of 4 cases. Ann Diagn Pathol 2010; 14: 317-20.

2. Xu AM, Xian ZH, Zhang SH, Chen XF. Intrahepatic cholangiocarcinoma arising in multiple bile duct hamartomas: report of two cases and review of the literature. Eur J Gastroenterol Hepatol 2009; 21: 580-4.

3. Nakanuma Y, Sato Y, Ikeda H, et al. Intrahepatic cholangiocarcinoma with predominant "ductal plate malformation" pattern: a new subtype. Am J Surg Pathol 2012; 36: 1629-35.

4. Nakanuma Y, Tsutsui A, Ren XS, Harada K, Sato Y, Sasaki M. What are the precursor and early lesions of peripheral intrahepatic cholangiocarcinoma? Int J Hepatol 2014; 2014: 805973.

5. Terada T. Combined hepatocellular-cholangiocarcinoma with stem cell features, ductal plate malformation subtype: a case report and proposal of a new subtype. Int J Clin Exp Pathol 2013; 6: 737-48.

6. Lindgren AG, Hansson G, Nilsson LA. Primary carcinoma arising in congenital liver in conjunction with miliary cholangiomatosis: report of case. Acta Pathol Microbiol Scand 1961; 52: 343-8.

7. Desmet VJ. Ductal plates in hepatic ductular reactions. Hypothesis and implications. III. Implications for liver pathology. Virchows Arch 2011; 458: 271-9.

8. Hasebe T, Sakamoto M, Mukai K, et al. Cholangiocarcinoma arising in bile duct adenoma with focal area of bile duct hamartoma. Virchows Arch 1995; 426: 209-13.

9. Pinho AC, Melo RB, Oliveira M, et al. Adenoma-carcinoma sequence in intrahepatic cholangiocarcinoma. Int J Surg Case Rep 2012; 3: 131-3.

10. Takahashi S, Takada K, Kawano Y, et al. Cholangiocarcinoma with bile duct adenoma and hamartoma-like lesion in the bile duct. Nihon Shokakibyo Gakkai Zasshi 2010; 107: 461-9. 